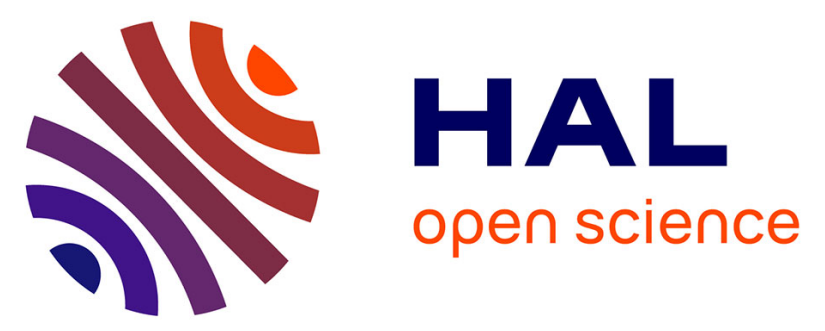

\title{
Faible efficacité des anti-TNF dans la spondyloarthrite axiale non-radiographique en l'absence de signes objectifs : étude rétrospective bicentrique
}

Céline Vidal, Cédric Lukas, Bernard Combe, Francis Berenbaum, Yves Marie Pers, Christian Jorgensen, Jérémie Sellam, Jacques Morel

\section{To cite this version:}

Céline Vidal, Cédric Lukas, Bernard Combe, Francis Berenbaum, Yves Marie Pers, et al.. Faible efficacité des anti-TNF dans la spondyloarthrite axiale non-radiographique en l'absence de signes objectifs: étude rétrospective bicentrique. Revue du Rhumatisme, 2019, 86, pp.275 - 282. 10.1016/j.rhum.2019.01.003 . hal-03486204

\section{HAL Id: hal-03486204 https://hal.science/hal-03486204}

Submitted on 20 Dec 2021

HAL is a multi-disciplinary open access archive for the deposit and dissemination of scientific research documents, whether they are published or not. The documents may come from teaching and research institutions in France or abroad, or from public or private research centers.
L'archive ouverte pluridisciplinaire HAL, est destinée au dépôt et à la diffusion de documents scientifiques de niveau recherche, publiés ou non, émanant des établissements d'enseignement et de recherche français ou étrangers, des laboratoires publics ou privés.

\section{(ㄷ)(1) $\$$}

Distributed under a Creative Commons Attribution - NonCommerciall 4.0 International 


\section{Faible efficacité des anti-TNF dans la spondyloarthrite axiale non- radiographique en l'absence de signes objectifs : une étude rétrospective bicentrique}

\section{Céline Vidal $^{1}$, Cédric Lukas ${ }^{1}$, Bernard Combe ${ }^{1}$, Francis Berenbaum ${ }^{2}$, Yves Marie Pers ${ }^{1}$, Christian Jorgensen ${ }^{1}$, Jérémie Sellam², Jacques Morel ${ }^{1}$}

${ }^{1}$ Service de Rhumatologie, Hôpital Lapeyronie, Université de Montpellier, 34295 Montpellier cedex 5, France

${ }^{2}$ AP-HP Hôpital St-Antoine, Service de Rhumatologie, DHU i2B, Inserm UMRS_938, 75012 Paris, France

Auteur correspondant : Céline Vidal, Service de Rhumatologie, Hôpital Lapeyronie, 371 Avenue du Doyen Gaston Giraud, 34295 Montpellier Cedex 5, France

celine_vidal@orange.fr 


\section{ABSTRACT}

Objectif : Comparer le taux de réponse BASDAI 50 aux anti-TNF dans la spondyloarthrite axiale (SpAax) en fonction de la présence ou non de signes objectifs de SpAax et rechercher les facteurs prédictifs de bonne réponse aux anti-TNF.

Méthodes : Les patients qui remplissaient le «bras clinique » des critères ASAS pour la SpAax et traités par anti-TNF entre janvier 2001 et septembre 2015 ont été retenus pour l'étude. Le premier groupe incluait les patients ayant au moins un signe objectif tel qu'une arthrite, dactylite, enthésite, uvéite, maladie inflammatoire digestive, CRP élevée ou sacroiliite radiologique et le second groupe incluait les sujets atteints d'une forme axiale nonradiographique de SpA (SpAax-nr) sans signes objectifs c'est-à-dire les patients souffrant de rachialgies inflammatoires avec une réponse adéquate aux AINS ou des antécédents familiaux de SpA. Le critère d'évaluation principal était l'efficacité des anti-TNF définie par la réponse BASDAI 50 à 3 mois. Les critères d'évaluation secondaires étaient la réponse BASDAI 50 sur un an et l'analyse des facteurs prédictifs de bonne réponse aux anti-TNF.

Résultats : Nous avons inclus 84 patients atteints de SpAax-nr sans signes objectifs et 84 patients atteints de SpAax avec des signes objectifs (48,2 \% SpAax-r et 52,8 \% SpAax-nr). La proportion de répondeurs BASDAI 50 était significativement plus élevée chez les patients ayant des signes objectifs comparativement aux sujets sans signes objectifs, à 3 mois (45,1\% contre $13,7 \%, \mathrm{p}<0,0001)$ et à chaque visite durant la première année de suivi $(61,9 \%$ contre 21,4\%, p < 0,0001). Dans une analyse multivariée, le surpoids/l'obésité et la sacro-iliite mise en évidence à l'IRM étaient des facteurs prédictifs de réponse respectivement négative et positive aux anti-TNF dans la population globale à 3 mois [OR =0,32, IC à $95 \%(0,11,0,96)$, $\mathrm{p}=0,041$ et $\mathrm{OR}=6,92$, IC à $95 \%(2,41,19,8), \mathrm{p}<0,0001$, respectivement $]$.

Conclusion : L'utilisation des anti-TNF doit être envisagée avec prudence dans la SpAax en l'absence de signes objectifs considérant que $13,7 \%$ seulement de ces patients étaient répondeurs BASDAI 50 à 3 mois.

Mots clés : Spondyloarthrite axiale, réponse aux anti-TNF, signes inflammatoires de spondyloarthrite, critères ASAS 


\section{Introduction}

La spondyloarthrite (SpA) regroupe un ensemble hétérogène de rhumatismes inflammatoires chroniques qui partagent certaines caractéristiques communes telles que la douleur inflammatoire axiale, l'atteinte périphérique et les manifestations extra-articulaires [1]. Les pathologies regroupées sous le concept de spondyloarthrite ont par ailleurs en commun une association génétique avec l'antigène de classe 1 HLA-B27 (Human Leucocyte Antigen B27) du complexe majeur d'histocompatibilité [2]. En 2009, l'ASAS (Assessment of SpondyloArthritis international Society) a élaboré de nouveaux critères de classification dans le but de détecter la $\mathrm{SpA}$ de manière plus précoce et de différencier les patients atteints de $\mathrm{SpA}$ en fonction de leur atteinte prédominante, axiale ou périphérique [3]. Les patients souffrant de lombalgies chroniques avec mise en évidence d'une sacro-iliite à la radiographie ou à l'IRM et présence d'au moins une manifestation évocatrice de spondyloarthrite, satisfaisaient au «bras imagerie » et les patients avec positivité du HLA-B27 et présence d'au moins deux manifestations de spondyloarthrite satisfaisaient au «bras clinique » des critères ASAS. Les patients atteints de SpA axiale (SpAax) ont été classés en deux groupes selon qu'il y avait présence ou non d'une sacro-iliite à la radiographie : SpAax radiographique (SpAax-r) incluant les patients ayant une sacro-iliite à la radiographie, et SpAax non-radiographique (SpAax-nr) incluant les patients avec ou sans sacro-iliite en IRM. L'efficacité des anti-TNF (Tumor Necrosis Factor) en l'absence de signes cliniques, biologiques ou radiologiques de SpAax reste méconnue en raison du faible nombre de patients inclus dans les études pivot mais compte tenu des effets secondaires graves des anti-TNF et de leur coût élevé, les indications de traitement nécessitent d'être précisées. Notre étude avait pour principal objectif de comparer le pourcentage de répondeurs BASDAI 50 aux anti-TNF à 3 mois dans la SpAax selon qu'il y avait présence ou absence de signes cliniques, radiologiques ou biologiques de SpAax. Les objectifs secondaires étaient de comparer la proportion de répondeurs sur un an dans les deux sous-groupes et d'évaluer les facteurs prédictifs de l'efficacité des anti-TNF.

\section{Méthodes}

\section{Population de l'étude}

Les données cliniques des patients atteints de SpAax d'après le diagnostic d'un rhumatologue expert, et remplissant le «bras clinique » des critères ASAS, donc porteurs du gène HLAB27, traités par un premier anti-TNF pendant au moins 3 mois consécutifs et âgés de plus de 16 ans à l'initiation de l'anti-TNF, ont été recueillies dans cette étude rétrospective bicentrique. Les anti-TNF ont été instaurés chez les patients atteints de SpAax ayant un score 
BASDAI $\geq 4 / 10$, en cas de réponse inadéquate ou d'intolérance aux AINS (AntiInflammatoires Non-Stéroïdiens) [4][5]. Les critères d'exclusion étaient la prise d'un antiTNF pour une raison autre que la SpA, telle qu'une maladie inflammatoire de l'intestin (MII) ou un psoriasis et l'absence de données cliniques, radiologiques et biologiques permettant d'établir le diagnostic de SpAax. Le score BASDAI à l'initiation des anti-TNF et au moins un autre score BASDAI durant le traitement étaient obligatoires.

Deux groupes ont été définis selon qu'il y avait présence ou non de signes objectifs de SpAax à l'instauration des anti-TNF. Le premier groupe incluait les patients ayant au moins un signe objectif, d'après le dossier médical, tel qu'une arthrite, dactylite, enthésite, uvéite, MII, CRP élevée ou une sacro-iliite à l'imagerie (à la radiographie ou en IRM). Le second groupe incluait les patients atteints de SpAax-nr sans aucun signe objectif. Les patients des deux groupes ont été appariés avec un ratio 1:1 pour le sexe, l'âge (différence maximum de 5 ans) et le type d'anti-TNF utilisé (récepteur ou anticorps monoclonal, puisque 7 patients sans signes objectifs et traités par anticorps monoclonal n'ont pas pu être appariés pour la même molécule anti-TNF). Le psoriasis n'a pas été considéré comme un signe objectif puisqu'il ne constitue pas un signe d'activité de la SpA [6][7][8]. De plus, la prévalence du psoriasis dans la population générale est élevée mais le nombre de patients atteints de psoriasis qui développent une SpA est faible [9]. L'inclusion des patients s'est déroulée de janvier 2001 à septembre 2015.

\section{Recueil des données}

Les données des patients ont été extraites des fichiers informatiques du Ministère de la Santé dans deux centres et les caractéristiques démographiques suivantes ont été collectées : âge à l'apparition de la maladie et à l'initiation de l'anti-TNF, statut tabagique en cours, indice de masse corporelle (IMC), consommation d'AINS, corticothérapie et traitements de fond antirhumatismaux (DMARD) conventionnels, état dépressif et prise d'antidépresseurs. Les caractéristiques cliniques, radiologiques et biologiques ayant servi au diagnostic de SpAax ont été recueillies et comprenaient la sacro-iliite radiographique, la sacro-iliite en IRM, le taux de CRP, l'IMC, la présence d'un psoriasis, d'une arthrite, uvéite, dactylite, enthésite, MII, les antécédents de réponse adéquate aux AINS, les antécédents familiaux de SpA, les antécédents de CRP élevée, de MII, de psoriasis, d'arthrite, d'uvéite, de dactylite et d'enthésite. Le score BASDAI à l'instauration des anti-TNF puis à 3, 6 et 12 mois a été relevé. Le délai et la raison motivant l'arrêt des anti-TNF ont été notés. En pratique clinique, le traitement par anti-TNF étant moins fréquent dans la SpAax-nr sans signes objectifs que dans la SpAax avec signes objectifs, nous avons tout d'abord recueilli les données des 84 patients du premier groupe puis 
les avons appariées à celles du groupe des SpAax avec signes objectifs. Compte tenu du caractère rétrospectif de notre étude qui reflète la réalité clinique, des données manquantes étaient attendues. C'est pourquoi, lorsque le BASDAI à 3 mois ne pouvait être officiellement évalué, et afin de conserver une puissance statistique maximale, nous avons considéré qu'il y avait réponse BASDAI 50 à 3 mois lorsqu'une nette amélioration était rapportée dans le dossier médical à la visite systématique des 3 mois et lorsque le patient était répondeur BASDAI 50 à 6 et/ou 12 mois de suivi. De la même manière, nous avons considéré l'absence de réponse BASDAI 50 à 3 mois lorsqu'aucune amélioration significative n'était spécifiée dans le rapport de la visite médicale et que le patient n'était pas répondeur BASDAI 50 à 6 et 12 mois. Cette étude a été menée conformément à la législation française et aux exigences du comité d'éthique local. Le consentement écrit a été obtenu préalablement à l'analyse génétique pour la recherche de l'allèle HLA-B27.

\section{Prescription des anti-TNF}

Les patients ont été traités par étanercept (ETN) à la dose de 25 milligrammes (mg) deux fois par semaine ou de $50 \mathrm{mg}$ une fois par semaine, ou par adalimumab (ADA) à une dose de $40 \mathrm{mg}$ toutes les 2 semaines, ou par infliximab (IFX) à raison de 3 à $5 \mathrm{mg} / \mathrm{kg}$ toutes les 6 à 8 semaines, ou par golimumab à la dose de $50 \mathrm{mg}$ une fois par mois, ou par certolizumab pegol (CZP) à une dose de $400 \mathrm{mg}$ toutes les 2 semaines au cours du premier mois, puis de $200 \mathrm{mg}$ toutes les deux semaines. Tous ces traitements sont indiqués dans la prise en charge de la SA et de la SpAax-nr (à l'exception de l'IFX) en cas de réponse inadéquate ou d'intolérance aux AINS et de score BASDAI $\geq 4 / 10[5]$.

\section{Critères d'évaluation}

L'efficacité des anti-TNF a été définie par la réponse BASDAI 50 à 3 mois pour le critère de jugement principal, et la réponse BASDAI 50 à chacune des visites trimestrielles effectuées durant la première année du suivi pour le critère de jugement secondaire.

\section{Analyse statistique}

À partir de l'étude ABILITY-1, durant laquelle les patients ayant des signes objectifs d'inflammation tels qu'une sacro-iliite en IRM ou une CRP initiale élevée, ont affiché une meilleure réponse à l'adalimumab comparativement au placebo [41\% (28/69) adalimumab contre $14 \%$ (10/73) placebo], contrairement aux patients sans sacro-iliite en IRM avec une 
CRP initiale normale [23\% (5/22) adalimumab contre $20 \%(4 / 20)$ placebo], nous avons supposé que le taux de répondeurs BASDAI 50 à 3 mois serait de $40 \%$ chez les patients avec des signes objectifs et de $20 \%$ chez les patients sans signes objectifs. Par conséquent, il était nécessaire d'inclure 79 patients dans chaque groupe pour mettre en évidence une différence statistiquement significative basée sur une erreur de type 1 de 0,05 avec une puissance de $80 \%$ [7]. Nous avons comparé la proportion de répondeurs et de non-répondeurs BASDAI 50 aux anti-TNF à 3 mois et pendant la première année dans les deux groupes en utilisant le test $\mathrm{du} \mathrm{chi}^{2}$. Les variables continues ont été rapportées en moyenne et écart-type (ET) puis comparées à l'aide du test-t de Student et les variables qualitatives ont été exprimées sous forme de fréquence et de pourcentage puis comparées au moyen du test du chi-carré. Le seuil de significativité statistique a été fixé à $\mathrm{p}<0,05$.

Une analyse multivariée testant les caractéristiques et leur association avec la réponse BASDAI 50 à 3 mois en analyse univariée $(\mathrm{p}<0,15)$ a ensuite été menée pour l'ensemble de la population (régression logistique utilisant la méthode de sélection des variables étape par étape).

Financement : Le travail décrit dans cet article n’a bénéficié d'aucun financement.

\section{Résultats}

\section{Caractéristiques des patients}

Quatre-vingt-quatre patients atteints de SpAax-nr sans signes objectifs et 84 patients atteints de SpAax avec au moins un signe objectif à l'instauration des anti-TNF, appariés pour le sexe, l'âge et le type d'anti-TNF ont été inclus. Les caractéristiques démographiques et pathologiques et l'activité de la maladie sont résumées dans le Tableau 1. Les patients étaient majoritairement de sexe féminin $(76,2 \%)$, avec un âge moyen \pm ET à l'initiation des antiTNF de 42,5 $\pm 11,1$ ans, une durée moyenne des symptômes de 10,6 \pm 8,5 ans et un IMC moyen de $25,5 \pm 5,4 \mathrm{~kg} / \mathrm{m}^{2}$. La proportion de fumeurs et de patients traités par antidépresseurs était similaire dans les deux groupes, et respectivement de 45,6\% et 20,2\%. La prise d'AINS, de corticoïdes et de DMARD était comparable entre le groupe SpAax avec signes objectifs et le groupe SpAax-nr sans signes objectifs [39/83 (47\%) contre 43/84 $(51,2 \%), 5 / 84(6 \%)$ contre $3 / 84(3,6 \%)$ et $6 / 84(7,1 \%)$ contre $3 / 84(3,6 \%)$ respectivement]. Le score BASDAI moyen à l'instauration des anti-TNF était similaire pour les deux groupes $(6,1 \pm 1,4$ dans le groupe des SpAax-nr sans signes objectifs et 5,6 $\pm 1,5$ dans le groupe des 
SpAax avec signes objectifs). Quatre-vingt-six patients $(51,2 \%)$ ont reçu un traitement par ETN et 82 patients $(48,8 \%)$ un traitement par ADA, IFX, CZP ou golimumab.

\section{Efficacité des anti-TNF}

La proportion de répondeurs BASDAI 50 à 3 mois était significativement plus élevée dans le groupe des SpAax avec signes objectifs comparativement au groupe des SpAax-nr sans signes objectifs [37/82 (45,1\% contre 11/80 (13,7\%) respectivement, p < 0,0001] (Figure 1). La proportion de répondeurs BASDAI 50 sur 1 an était également significativement plus élevée dans le groupe des SpAax avec signes objectifs comparativement au groupe des SpAax-nr sans signes objectifs [52/84 (61,9\%) contre 18/84 (21,4\%) respectivement, p < 0,0001]

(Figure 1). À noter que 14 patients supplémentaires du groupe SpAax avec signes objectifs et 6 patients supplémentaires du groupe SpAax-nr sans signes objectifs étaient répondeurs BASDAI 50 à 6 mois parmi les 84 patients de chaque groupe pour lesquels ces données étaient disponibles.

Un patient atteint de SpAax et 1 patient atteint de SpAax-nr ont été considérés répondeurs BASDAI 50 à 3 mois compte tenu de la nette amélioration clinique rapportée dans leur dossier médical (mais sans mesure du score BASDAI disponible) et du fait qu'ils étaient répondeurs BASDAI 50 à 6 et/ou 12 mois, et ce, malgré l'absence du score BASDAI 50 à 3 mois.

\section{Facteurs prédictifs de l'efficacité des anti-TNF :}

Analyse univariée :

Pour l'ensemble de la population, les facteurs associés à la réponse BASDAI 50 à 3 mois et sur 1 an par analyse univariée sont résumés dans le Tableau 2. Les facteurs prédictifs de bonne réponse à 3 mois étaient l'existence d'une sacro-iliite radiographique, d'une sacro-iliite en IRM, un taux élevé de CRP et un score BASDAI moyen inférieur à l'instauration des antiTNF. Les répondeurs BASDAI 50 étaient plus jeunes et moins nombreux à avoir un IMC $\geq$ $25 \mathrm{~kg} / \mathrm{m}^{2}$ comparativement aux non-répondeurs, mais cette différence n'était pas statistiquement significative. Les facteurs prédictifs de bonne réponse sur 1 an étaient l'existence d'une sacro-iliite radiographique, d'une sacro-iliite en IRM, une CRP élevée, des antécédents de CRP élevée et un score BASDAI moyen inférieur à l'instauration des antiTNF. Le facteur prédictif de mauvaise réponse sur un an était un IMC $\geq 25 \mathrm{~kg} / \mathrm{m}^{2}$. 
Parmi les patients du groupe SpAax-nr sans signes objectifs, le surpoids/l'obésité (IMC $\geq$ $25 \mathrm{~kg} / \mathrm{m}^{2}$ ) était un facteur prédictif négatif supplémentaire de réponse BASDAI 50 à 3 mois et sur un an [Annexe A, Tableaux S1-S2 ; voir le matériel complémentaire accompagnant la version en ligne de cet article].

Parmi les patients du groupe SpAax avec signes objectifs, la prise d'AINS était un facteur prédictif positif de réponse BASDAI 50 à 3 mois et sur un an. Les répondeurs BASDAI 50 étaient plus souvent des hommes, avec une significativité statistique à 12 mois mais pas à 3 mois [Annexe A, Tableaux S1-S2].

Analyse multivariée :

Seuls 97 patients sur 168 ont été inclus dans l'analyse en raison de la distribution aléatoire des données manquantes. Le surpoids/l'obésité et la sacro-iliite à l'IRM étaient des facteurs prédictifs respectivement négatifs et positifs de l'efficacité des anti-TNF dans cette population à 3 mois $[\mathrm{OR}=0,32$, IC à $95 \%(0,11,0,96), \mathrm{p}=0,041$ et $\mathrm{OR}=6,92, \mathrm{IC}$ à $95 \%(2,41,19,82)$, $\mathrm{p}<0,0001$, respectivement].

\section{Taux de maintien thérapeutique et effets secondaires des anti-TNF}

Le taux d'arrêt des anti-TNF sur un an était significativement plus élevé dans le groupe des SpAax-nr sans signes objectifs comparativement au groupe des SpAax avec signes objectifs [53/80 (66,2\%) contre 31/75 (41,3\%), p <0,001] (Figure 2). La cause d'arrêt la plus fréquente dans les deux groupes était l'inefficacité thérapeutique [43/53 (81,1\%) dans le groupe SpAax-nr sans signes objectifs contre 22/31 (71\%) dans le groupe SpAax avec signes objectifs].

La survenue d'effets secondaires était comparable entre le groupe SpAax-nr sans signes objectifs et le groupe SpAax avec signes objectifs [13/84 (15,5\%) contre 20/80 (25\%), p $=0,092]$ et les infections sans gravité représentaient la majorité des effets secondaires rapportés.

\section{Discussion}

L'objectif de cette étude était d'examiner l'évolution des scores BASDAI après l'instauration des anti-TNF en l'absence de signes cliniques, biologiques ou radiologiques de SpAax. Nos résultats ont révélé un taux de répondeurs BASDAI 50 significativement plus faible dans la SpAax-nr sans signes objectifs comparativement à la SpAax avec signes objectifs à 3 mois $(13,7 \%$ contre $45,1 \%)$ et pendant la première année $(21,4 \%$ contre $61,9 \%)$. Par ailleurs, le surpoids ou l'obésité (IMC $\geq 25 \mathrm{~kg} / \mathrm{m}^{2}$ ) ont été rapportés comme étant des facteurs prédictifs 
négatifs de réponse BASDAI 50 à 3 mois et pendant la première année dans la SpAax-nr sans signes objectifs.

Dans la plupart des études évaluant l'efficacité des traitements de la SpAax-r et de la SpAaxnr, les taux de réponse ASAS 40 et ASAS 20 sont les principaux paramètres utilisés. Nous avons choisi dans notre étude d'utiliser la réponse BASDAI 50 pour évaluer l'efficacité des anti-TNF, en raison du caractère rétrospectif de notre étude et de la non-disponibilité des scores ASAS 40 et ASAS 20. C'est pourquoi la comparaison de nos résultats avec ceux d'autres études pourrait être considérée comme non valide mais les réponses BASDAI 50 et ASAS 40 sont très proches et évoluent dans des proportions semblables ce qui rend les comparaison relativement acceptables [10][11]. Dans notre étude, le taux de répondeurs BASDAI 50 observé dans le groupe SpAax-nr sans signes objectifs est comparable à celui des répondeurs ASAS 40 rapporté dans la SpAax-nr sans sacro-iliite en IRM ni CRP élevée, mais également similaire au taux de répondeurs chez les sujets ayant reçu un placebo au cours de différentes études [7][12]. La faible efficacité des anti-TNF retrouvée dans le groupe SpAaxnr sans signes objectifs résulte probablement d'un mauvais diagnostic comme suggéré par une étude qui a rapporté un pourcentage de $13,6 \%$ des patients avec rachialgies inflammatoires et répondant aux critères ASAS mais n'ayant pas reçu le diagnostic de SpAax par les rhumatologues experts. Dans cette étude de Lin Z. et al., $20 \%$ des sujets souffraient de pathologies dégénératives telles qu'une arthrite lombaire ou une hernie discale [13]. Ces résultats ont été confirmés par une récente étude portant sur la validité des critères ASAS à 4 ans, et qui là encore a mis en évidence une erreur de diagnostic chez $12 \%$ des patients satisfaisant au bras clinique des critères ASAS [14]. En effet, les critères ASAS ont été développés pour la classification des patients au cours d'essais cliniques et d'études épidémiologiques mais ne devraient pas être utilisés comme critères diagnostiques, l'avis des experts restant le «gold standard». De plus, les patients sans signes objectifs avaient soit une bonne réponse aux AINS soit une histoire familiale de $\mathrm{SpA}$, mais ces deux caractéristiques n'ont pas été vérifiées car il s'agit d'éléments rapportés par le patient susceptibles d'entraîner une erreur de classification. En effet, les sujets avec histoire familiale de SpA étaient plus fréquemment que les autres mauvais répondeurs aux anti-TNF et seulement 43/63 patients sans signes objectifs avec des antécédents de bonne réponse aux AINS étaient sous traitement par AINS, ce qui renforce cette hypothèse. Cependant, dans notre étude, tous les patients avaient fait l'objet d'une évaluation par un rhumatologue expert et avaient reçu le diagnostic de SpAax au moment de l'instauration des anti-TNF. Cette différence d'efficacité des anti- 
TNF entre les deux groupes est peut-être due à un biais de classification concernant la sévérité et l'activité de la maladie puisqu'une CRP élevée au départ et une sacro-iliite en IRM sont associées à une activité élevée de la maladie et une meilleure réponse aux anti-TNF.

Toutefois, l'activité de la maladie à l'instauration des anti-TNF, comme évaluée par le score BASDAI, était similaire entre les deux groupes mais il est à noter que le BASDAI n'est pas spécifique à la SpA, puisque des scores BASDAI similaires ou plus élevés peuvent être observés chez des patients atteints d'autres pathologies telles que la fibromyalgie. Une autre cause du manque d'efficacité des anti-TNF dans la SpAax-nr sans signes objectifs pourrait être l'association avec la fibromyalgie. En effet, la prévalence de la fibromyalgie peut atteindre $25 \%$ chez les patients souffrant de maladies rhumatismales inflammatoires et une récente étude française a rapporté une proportion de 21,4\% de patients atteints de SpAax-nr, sans sacro-iliite à l'IRM ni CRP élevée, pour lesquels le diagnostic de fibromyalgie était également posé [15][16]. De plus, dans environ 20 à $30 \%$ des cas, les patients atteints de fibromyalgie souffrent de rachialgies inflammatoires ce qui rend plus difficile la distinction entre fibromyalgie et SpAax-nr [17]. C'est pourquoi, une fibromyalgie concomitante peut avoir été à l'origine de toutes les plaintes chez certains patients et pourrait expliquer le sexratio inhabituel observé. Dans une précédente étude, les patients atteints de SpAax avec fibromyalgie étaient significativement plus nombreux à recevoir un traitement antidépresseur comparativement aux patients atteints de SpAax sans fibromyalgie, bien que l'utilisation des antidépresseurs ne soit pas un substitut à la présence de la fibromyalgie [16]. En raison du caractère rétrospectif de notre étude, la présence d'une fibromyalgie n'a pas été systématiquement évaluée. Cependant, le nombre de patients traités par antidépresseurs n'était pas significativement plus élevé dans le groupe SpAax-nr sans signes objectifs (25,3\% contre $15,5 \%$ des patients ayant des signes objectifs, $\mathrm{p}=0,086$ ) mais ce résultat est probablement dû à un manque de puissance statistique puisque la tendance semble similaire. Pour finir, les patients atteints de SpAax-nr avec une fibromyalgie associée pourraient être considérés comme ayant une forme « enthésopathique » de SpA (cependant sans enthésite objectivement définie d'après les données d'imagerie disponibles). Très peu d'études ont évalué l'efficacité des anti-TNF dans la SpA avec enthésite mais aucune amélioration clinique significative n'a été relevée chez un petit nombre de patients traités par ADA [18]. Par ailleurs, chez les patients atteints de SpAax-nr inclus dans l'étude ABILITY 1, avec une sacro-iliite à l'IRM dans seulement $50 \%$ des cas, aucune amélioration du score MASES (Maastricht Ankylosing Spondylitis Enthesitis Score) n'a été constatée sous traitement ADA [7]. Inversement, dans la SpAax-nr avec respectivement $80 \%$ et $66 \%$ de sacro-iliites à 
l'IRM, le score MASES a été significativement amélioré sous traitement par ETA et golimumab [11][12]. Ces résultats soulignent l'inefficacité des anti-TNF dans la SpAax-nr avec enthésopathie en l'absence de signes objectifs. Cependant, nous n'avons pas été en mesure d'évaluer cette hypothèse dans notre étude rétrospective car nous ne disposions pas du score MASES. La mauvaise classification peut être due uniquement au hasard puisque la prévalence de la $\mathrm{SpA}$ en France était estimée à $0,3 \%$ et la prévalence des lombalgies inflammatoires chroniques et de la fibromyalgie avoisinait respectivement 7,5\% et $5 \%$ ce qui conduit à un pourcentage d'adultes souffrant conjointement de ces deux conditions s'élevant à $0,37 \%[19][20][21]$.

Notre étude confirme les données déjà publiées concernant les facteurs prédictifs de réponse aux anti-TNF [22][23]. En effet, dans une analyse univariée portant sur la population totale, les facteurs prédictifs de bonne réponse aux anti-TNF à 3 mois et sur un an étaient une sacroiliite radiographique, une sacro-iliite à l'IRM, une CRP élevée et un faible score BASDAI initial moyen. L'IMC $\geq 25 \mathrm{~kg} / \mathrm{m}^{2}$ était un facteur prédictif négatif à un an dans la population totale mais sans significativité statistique à 3 mois pour l'ensemble de la population (p =0,054). De la même manière, le surpoids ou l'obésité étaient des facteurs prédictifs de mauvaise réponse aux anti-TNF à 3 mois et sur un an pour le groupe SpAax-nr sans signes objectifs. L'IMC élevé comme facteur prédictif de mauvaise réponse aux anti-TNF dans la spondylarthrite ankylosante (SA) a déjà été rapporté au cours de deux études, en particulier chez les patients traités par IFX, la cause étant probablement l'administration de doses insuffisantes [24][25].

Notre étude a mis en évidence une proportion plus élevée de répondeurs BASDAI 50 dans le groupe de patients ayant un faible score BASDAI initial. Certaines études ont rapporté une association entre le score BASDAI initial élevé et l'efficacité des anti-TNF mais il s'agissait principalement d'études randomisées cas-témoins portant uniquement sur les formes radiographiques de SpA axiale [26]. Dans notre étude, le score BASDAI moyen élevé pourrait être attribuable à une fibromyalgie concomitante chez plusieurs patients du groupe SpAax-nr sans signes objectifs. En effet, la fibromyalgie est associée à un score BASDAI plus élevé comparativement aux patients sans fibromyalgie [16][27]. Dans notre étude, cette hypothèse est confortée par le score BASDAI moyen initial plus élevé dans le groupe SpAaxnr sans signes objectifs même si cette différence n'a pas été considérée comme significative [6,07 $(1,44)$ contre 5,65 (1,52), $\mathrm{p}=0,069]$.

Comme rapporté dans de précédentes études, on observait dans le groupe SpAax avec signes 
objectifs une tendance vers une proportion plus importante de répondeurs BASDAI 50 chez les hommes à 3 mois, mais cette différence n'a pas été considérée comme statistiquement significative [22]. Dans notre étude, le tabagisme n'a pas été identifié comme facteur prédictif de réponse aux anti-TNF. À ce jour, seule une étude a rapporté le tabagisme comme étant un facteur prédictif de mauvaise réponse aux anti-TNF chez des patients ayant une CRP élevée [28]. D’autres études seront nécessaires pour évaluer l'impact du tabagisme sur la réponse thérapeutique. Dans notre étude, dans le groupe SpAax sans signes objectifs, la prise d'AINS au départ - pouvant traduire un bénéfice thérapeutique persistant du point de vue du patient et donc une maladie active - a été associée à une plus grande proportion de répondeurs BASDAI 50 à 3 mois. Une seule étude a montré que la consommation d'AINS n'était pas un facteur prédictif de réponse à l'IFX dans la SA et d'autres études seront nécessaires pour évaluer l'impact des AINS sur l'efficacité des anti-TNF [29].

Notre étude a mis en évidence un taux de réponse BASDAI 50 plus élevé chez les patients ayant une sacro-iliite radiographique. Plusieurs études ont montré une efficacité similaire des anti-TNF dans la SA et SpAax-nr mais la majorité des patients SpAax-nr inclus avaient une sacro-iliite à l'IRM et/ou une CRP élevée et ces deux paramètres sont des facteurs prédictifs positifs importants de l'efficacité des anti-TNF. Par conséquent, l'identification de la sacroiliite radiographique comme facteur prédictif est improbable [8][30][31][32]. Dans une analyse multivariée, la sacro-iliite radiographique et l'élévation de la CRP n'ont pas été identifiées comme facteurs prédictifs significatifs de réponse BASDAI 50 aux anti-TNF, mais le nombre élevé de données manquantes et la colinéarité importante entre ces deux caractéristiques (la plupart des patients ayant une CRP anormale avaient également une IRM positive) ne permettent pas d'estimer précisément leur impact respectif sur la réponse thérapeutique.

Les antécédents de signes objectifs n'étaient pas considérés comme facteurs prédictifs de bonne réponse aux anti-TNF dans notre étude, à l'exception des antécédents de CRP anormalement élevée pour la réponse sur un an. Cependant, tous les patients ayant une CRP anormalement élevée au moment de l'étude avaient également des antécédents de CRP élevée ce qui, là encore, limite fortement la possibilité d'en analyser l'impact respectif.

Le maintien thérapeutique des anti-TNF à un an était significativement plus marqué dans le groupe SpAax avec signes objectifs que dans le groupe SpAax-nr sans signes objectifs, en raison principalement d'une meilleure réponse thérapeutique. Ces résultats corroborent ceux 
d'une étude suédoise selon laquelle les patients satisfaisant au bras clinique des critères ASAS, sans sacro-iliite à l'IRM, affichaient un taux de maintien des anti-TNF de seulement $30 \%$ à 12 mois par comparaison au taux de maintien thérapeutique de $74 \%$ relevé chez les patients répondant au bras imagerie [33].

Cette étude souligne la difficulté d'interrompre un traitement inefficace après son instauration. En effet, malgré l'absence de réponse thérapeutique significative chez certains patients, la prescription a parfois été renouvelée. À trois mois, seuls 11/80 patients du groupe SpAax-nr sans signes objectifs ont été classés comme répondeurs et le traitement anti-TNF a pourtant été renouvelé chez 64/83 patients, et à un an, seulement 18/84 patients du groupe SpAax-nr sans signes objectifs ont été classés comme répondeurs, mais le traitement par antiTNF a là encore été renouvelé chez 27/80 patients.

Notre étude présente certaines limites. Compte tenu de son caractère rétrospectif, certaines données manquantes ont pu affecter la puissance statistique de nos résultats mais la proportion des données manquantes était globalement inférieure à $25 \%$ et tous les facteurs pertinents - définis a priori - étaient disponibles car obligatoires pour l'inclusion des patients dans la présente étude. La SpA peut avoir été diagnostiquée à tort à la suite d'une surinterprétation d'un œdème osseux de faible volume au niveau de l'articulation sacroiliaque comme démontré dans une étude au cours de laquelle 107/691 (15,5\%) patients suspectés d'être atteints de SpA présentaient des modifications structurales du rachis (modifications dégénératives ou anomalies passagères) avec un œdème osseux adjacent [34]. Dans l'étude Ability-1, 3/16 patients avec HLA-B27 négatif et sacro-iliite à l'IRM et traités par adalimumab (19\%) ont obtenu une réponse ASAS 40, contre 3/24 patients traités par placebo $(13 \%)(p=0,42)$ [7]. Par ailleurs, dans une récente étude, $21 \%$ des patients souffrant de rachialgies inflammatoires chroniques satisfaisaient aux critères ASAS avec une sacroiliite à l'IRM mais un faible score d'œdème osseux était associé à chacune des caractéristiques cliniques des critères ASAS [35]. Cependant, dans notre étude, en cas de doute, les clichés IRM ont été analysés par un radiologue, spécialiste en imagerie musculosquelettique .

Nous n'avons pas pu nous appuyer sur le taux de réponse ASAS 40 ou 20 pour évaluer l'efficacité des anti-TNF mais le taux de réponse BASDAI 50 est souvent utilisé comme critère secondaire dans les études et nous a permis de comparer nos résultats avec les données de la littérature. Aucun des patients de notre étude n'avait d'enthésite objective ou 
d'antécédents d'enthésite objective selon les éléments d'imagerie disponibles et nos résultats ne peuvent donc pas être généralisés aux patients ayant ce paramètre comme seul signe objectif.

En conclusion nous insistons sur le fait que de nombreux patients sans signes objectifs avaient des plaintes qui n'étaient probablement pas attribuables à la SpAax ou ont fait l'objet d'un diagnostic erroné de SpAax-nr. C'est pourquoi, l'utilisation des anti-TNF devrait être exclue en l'absence de signes objectifs au moment d'instaurer le traitement et ne devrait pas servir à tester la validité du diagnostic de SpA lorsqu'il y a absence de signes objectifs.

Approbation éthique et consentement : cette étude rétrospective a été menée conformément à la législation française et n'a pas nécessité l'approbation d'un comité d'éthique. Le consentement écrit a été obtenu préalablement à l'analyse génétique de l'allèle HLA-B27.

Disponibilité des données et du matériel : Toutes les données produites ou analysées durant cette étude sont incluses dans l'article publié [et dans la documentation supplémentaire].

Conflits d'intérêts : Les auteurs ne déclarent aucun conflit d'intérêt.

Contributions des auteurs : CV a contribué de manière substantielle à la conception de l'étude et à l'interprétation des données, a participé à l'élaboration et à la révision du contenu intellectuel de l'article, a approuvé la version finale pour publication et engage sa responsabilité concernant tous les aspects de ce travail. CL a contribué de manière substantielle à la conception de l'étude et à l'interprétation des données, a supervisé l'analyse statistique, a participé à la révision du contenu intellectuel de l'article, a approuvé la version finale pour publication et engage sa responsabilité concernant tous les aspects de ce travail. $\mathrm{BC}$ a contribué de manière substantielle à la conception de l'étude et à l'interprétation des données, a participé à la révision du contenu intellectuel de l'article, a approuvé la version finale pour publication et engage sa responsabilité concernant tous les aspects de ce travail. FB a contribué de manière substantielle à la conception de l'étude et à l'interprétation des données, a participé à la révision du contenu intellectuel de l'article, a approuvé la version 
finale pour publication et engage sa responsabilité concernant tous les aspects de ce travail. YMP a contribué de manière substantielle à la conception de l'étude et à l'interprétation des données, a participé à la révision du contenu intellectuel de l'article, a approuvé la version finale pour publication et engage sa responsabilité concernant tous les aspects de ce travail. CJ a contribué de manière substantielle à la conception de l'étude et à l'interprétation des données, a participé à la révision du contenu intellectuel de l'article, a approuvé la version finale pour publication et engage sa responsabilité concernant tous les aspects de ce travail. JS a contribué de manière substantielle à la conception de l'étude et à l'interprétation des données, a participé à la révision du contenu intellectuel de l'article, a approuvé la version finale pour publication et engage sa responsabilité concernant tous les aspects de ce travail. JM a contribué de manière substantielle à la conception de l'étude et à l'interprétation des données, a participé à la révision du contenu intellectuel de l'article, a approuvé la version finale pour publication et engage sa responsabilité concernant tous les aspects de ce travail. Tous les auteurs ont lu et approuvé la version finale de l'article.

Remerciements : Les auteurs remercient le Dr P. Leblay, le Dr C. Beauvais et le Dr N. Courvoisier pour leur participation au recueil des données des patients.

Annexe A. Matériel complémentaire

Du matériel complémentaire (Tableaux S1-S2) accompagnant cet article est disponible sur ... 


\section{Références}

[1] Dougados M, Baeten D. Spondyloarthritis. Lancet Lond Engl 2011;377:2127-37.

[2] Rudwaleit M. New approaches to diagnosis and classification of axial and peripheral spondyloarthritis. Curr Opin Rheumatol 2010;22:375-80.

[3] Rudwaleit M, van der Heijde D, Landewé R, et al. The development of Assessment of SpondyloArthritis international Society classification criteria for axial spondyloarthritis (part II): validation and final selection. Ann Rheum Dis 2009;68:77783.

[4] Smolen JS, Braun J, Dougados M, et al. Treating spondyloarthritis, including ankylosing spondylitis and psoriatic arthritis, to target: recommendations of an international task force. Ann Rheum Dis 2014;73:6-16.

[5] Ward MM, Deodhar A, Akl EA, et al. American College of Rheumatology/Spondylitis Association of America/Spondyloarthritis Research and Treatment Network 2015 Recommendations for the Treatment of Ankylosing Spondylitis and Nonradiographic Axial Spondyloarthritis. Arthritis Rheumatol Hoboken NJ 2016;68:282-98.

[6] Haibel H, Rudwaleit M, Listing J, et al. Efficacy of adalimumab in the treatment of axial spondylarthritis without radiographically defined sacroiliitis: results of a twelveweek randomized, double-blind, placebo-controlled trial followed by an open-label extension up to week fifty-two. Arthritis Rheum 2008;58:1981-91.

[7] Sieper J, van der Heijde D, Dougados M, et al. Efficacy and safety of adalimumab in patients with non-radiographic axial spondyloarthritis: results of a randomised placebocontrolled trial (ABILITY-1). Ann Rheum Dis 2013;72:815-22.

[8] Landewe R, Braun J, Deodhar A, et al. Efficacy of certolizumab pegol on signs and symptoms of axial spondyloarthritis including ankylosing spondylitis: 24-week results of a double-blind randomised placebo-controlled Phase 3 study. Ann Rheum Dis 2014;73:39-47.

[9] Rouzaud M, Sevrain M, Villani AP, et al. Is there a psoriasis skin phenotype associated with psoriatic arthritis? Systematic literature review. J Eur Acad Dermatol Venereol JEADV 2014;28 Suppl 5:17-26.

[10] Ciurea A, Scherer A, Exer P, et al. Tumor necrosis factor $\alpha$ inhibition in radiographic and nonradiographic axial spondyloarthritis: results from a large observational cohort. Arthritis Rheum 2013;65:3096-106.

[11] Dougados M, van der Heijde D, Sieper J, et al. Symptomatic Efficacy of Etanercept and Its Effects on Objective Signs of Inflammation in Early Nonradiographic Axial Spondyloarthritis: A Multicenter, Randomized, Double-Blind, Placebo-Controlled Trial: Efficacy of Etanercept in Nonradiographic Axial SpA. Arthritis Rheumatol 2014;66:2091-102.

[12] Sieper J, van der Heijde D, Dougados M, et al. A randomized, double-blind, placebo-controlled, sixteen-week study of subcutaneous golimumab in patients with active nonradiographic axial spondyloarthritis. Arthritis Rheumatol Hoboken NJ 2015;67:2702-12.

[13] Lin Z, Liao Z, Huang J, Jin O, Li Q, Li T, et al. Evaluation of Assessment of Spondyloarthritis International Society classification criteria for axial spondyloarthritis in Chinese patients with chronic back pain: results of a 2-year follow-up study. Int J Rheum Dis 2014;17:782-9.

[14] Sepriano A, Landewé R, van der Heijde D, et al. Predictive validity of the ASAS classification criteria for axial and peripheral spondyloarthritis after follow-up in the 
ASAS cohort: a final analysis. Ann Rheum Dis 2016;75:1034-42.

[15] Haliloglu S, Carlioglu A, Akdeniz D, Karaaslan Y, Kosar A. Fibromyalgia in patients with other rheumatic diseases: prevalence and relationship with disease activity. Rheumatol Int 2014;34:1275-80.

[16] Bello N, Etcheto A, Béal C, Dougados M, Moltó A. Evaluation of the impact of fibromyalgia in disease activity and treatment effect in spondyloarthritis. Arthritis Res Ther 2016;18:42.

[17] Roussou E, Ciurtin C. Clinical overlap between fibromyalgia tender points and enthesitis sites in patients with spondyloarthritis who present with inflammatory back pain. Clin Exp Rheumatol 2012;30:24-30.

[18] Maksymowych WP, Mallon C, Morrow S, et al. Development and validation of the Spondyloarthritis Research Consortium of Canada (SPARCC) Enthesitis Index. Ann Rheum Dis 2009;68:948-53.

[19] Roux CH, Saraux A, Le Bihan E, et al. Rheumatoid arthritis and spondyloarthropathies: geographical variations in prevalence in France. J Rheumatol 2007;34:117-22.

[20] Depont F, Hunsche E, Abouelfath A, et al. Medical and non-medical direct costs of chronic low back pain in patients consulting primary care physicians in France. Fundam Clin Pharmacol 2010;24:101-8.

[21] Bannwarth B, Blotman F, Roué-Le Lay K, Caubère J-P, André E, Taïeb C.

Fibromyalgia syndrome in the general population of France: a prevalence study. Jt Bone Spine Rev Rhum 2009;76:184-7.

[22] Gremese E, Bernardi S, Bonazza S, et al. Body weight, gender and response to TNF- $\alpha$ blockers in axial spondyloarthritis. Rheumatol Oxf Engl 2014;53:875-81.

[23] Maas F, Arends S, van der Veer E, et al. Obesity Is Common in Axial Spondyloarthritis and Is Associated with Poor Clinical Outcome. J Rheumatol 2016;43:383-7.

[24] Ottaviani S, Allanore Y, Tubach F, et al. Body mass index influences the response to infliximab in ankylosing spondylitis. Arthritis Res Ther 2012;14:R115.

[25] Gremese E, Bernardi S, Bonazza S, et al. Body weight, gender and response to TNF- $\alpha$ blockers in axial spondyloarthritis. Rheumatol Oxf Engl 2014;53:875-81.

[26] Rudwaleit M. Prediction of a major clinical response (BASDAI 50) to tumour necrosis factor blockers in ankylosing spondylitis. Ann Rheum Dis 2004;63:665-70.

[27] Lukas C, Dougados M, Combe B. Factors associated with a bad functional prognosis in early inflammatory back pain: results from the DESIR cohort. RMD Open 2016;2:e000204.

[28] Ciurea A, Scherer A, Weber U, et al. Impaired response to treatment with tumour necrosis factor inhibitors in smokers with axial spondyloarthritis. Ann Rheum Dis 2016;75:532-9.

[29] Lorenzin M, Ortolan A, Frallonardo P, Oliviero F, Punzi L, Ramonda R. Predictors of response and drug survival in ankylosing spondylitis patients treated with infliximab. BMC Musculoskelet Disord 2015;16:166.

[30] Barkham N, Keen HI, Coates LC, et al. Clinical and imaging efficacy of infliximab in HLA-B27-Positive patients with magnetic resonance imaging-determined early sacroiliitis. Arthritis Rheum 2009;60:946-54.

[31] Song IH, Weiß A, Hermann K-GA, et al. Similar response rates in patients with ankylosing spondylitis and non-radiographic axial spondyloarthritis after 1 year of treatment with etanercept: results from the ESTHER trial. Ann Rheum Dis 2013;72:8235 . 
[32] Poddubnyy D, Sieper J. Similarities and differences between nonradiographic and radiographic axial spondyloarthritis: a clinical, epidemiological and therapeutic assessment. Curr Opin Rheumatol 2014;26:377-83.

[33] Gulfe A, Kapetanovic M, Kristensen L. Efficacy and drug survival of anti-tumour necrosis factor-alpha therapies in patients with non-radiographic axial

spondyloarthritis: an observational cohort study from Southern Sweden. Scand J Rheumatol 2014;43:493-7.

[34] Jans L, Van Praet L, Elewaut D, et al. MRI of the SI joints commonly shows noninflammatory disease in patients clinically suspected of sacroiliitis. Eur J Radiol 2014;83:179-84.

[35] Arnbak B, Grethe Jurik A, et al. Associations Between Spondyloarthritis Features and Magnetic Resonance Imaging Findings: A Cross-Sectional Analysis of 1,020 Patients With Persistent Low Back Pain. Arthritis Rheumatol Hoboken NJ 2016;68:892-900. 
Figure 1 : Taux de réponse BASDAI 50 à 3 mois et à 3, 6 ou 12 mois.

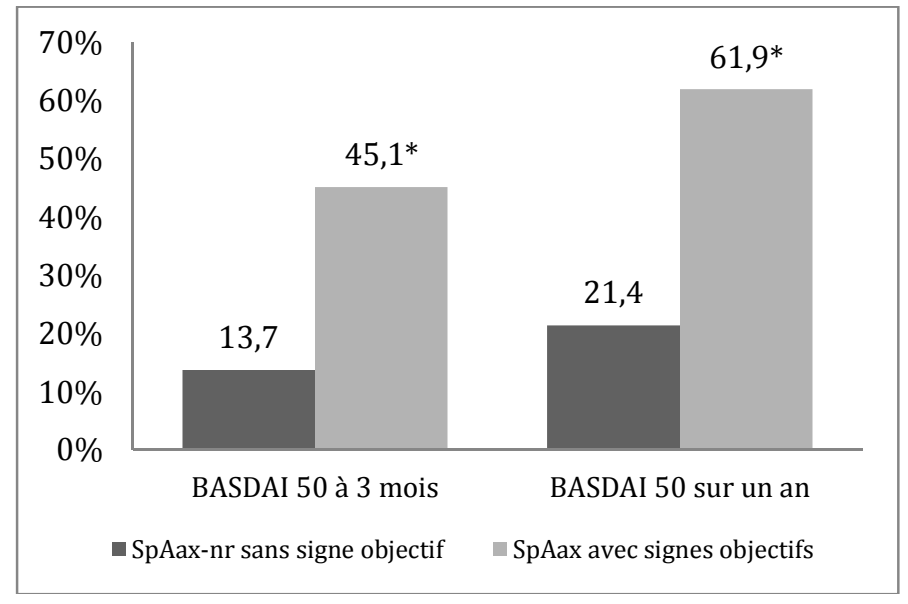

* $\mathrm{p}<0,0001$ pour la comparaison de la réponse thérapeutique entre la SpAax avec des signes objectifs d'inflammation et la SpAax-nr sans signes objectifs d'inflammation.

$\S \mathrm{n}=154$ (73 SpAax-nr sans signes objectifs et 81 SpAax avec des signes objectifs)

SpAax : spondyloarthrite axiale, BASDAI : Bath Ankylosing Spondylitis Disease Activity Index, SpAax-nr : spondyloarthrite axiale non-radiographique. 
Figure 2 : Arrêt des anti-TNF à 3 mois, 6 mois et sur un an

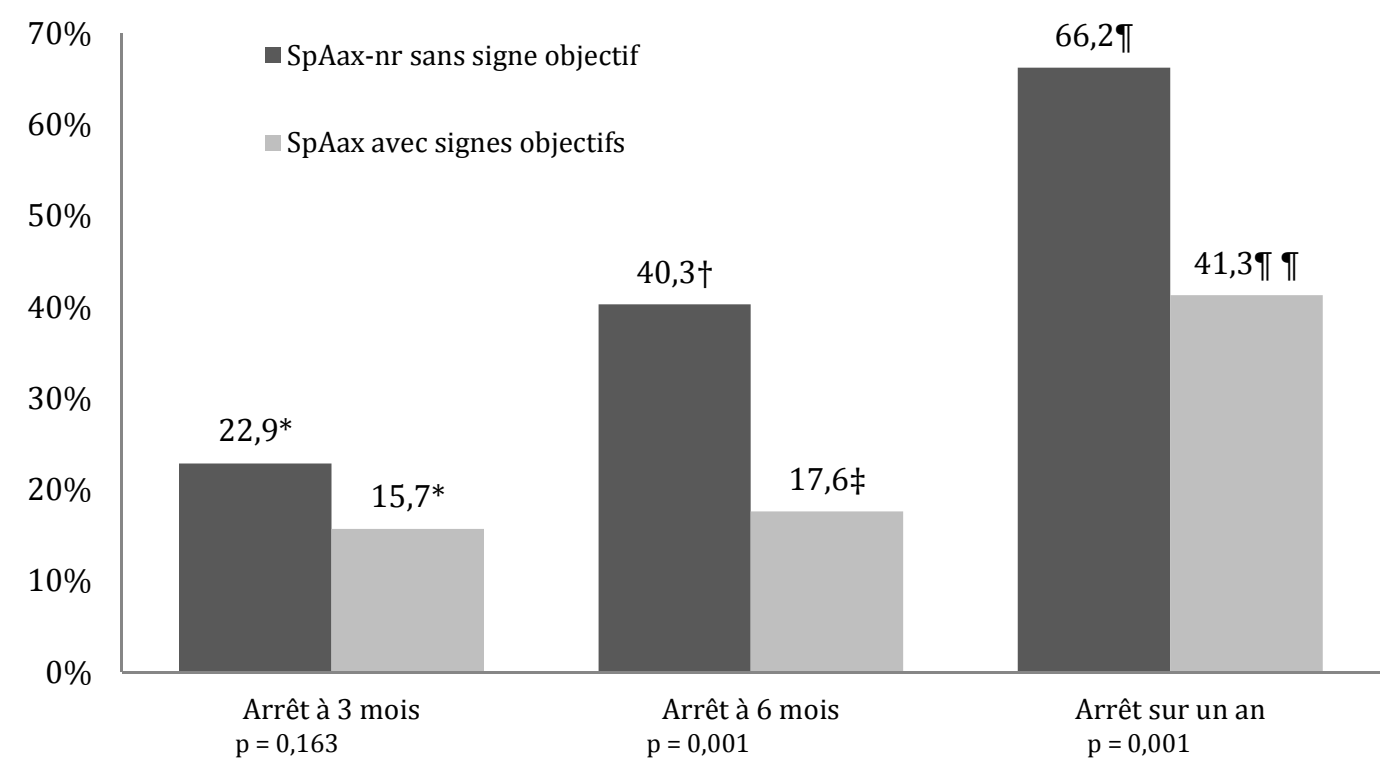

$* \mathrm{n}=83 ; \dagger \mathrm{n}=62 ; \ddagger \mathrm{n}=68 ;$ II $\mathrm{n}=80 ;$ III $\mathrm{n}=75$; SpAax $:$ spondyloarthrite axiale, SpAax-nr : spondyloarthrite axiale non-radiographique. 
Tableau 1 : Caractéristiques démographiques et pathologiques à l'instauration des anti-TNF.

\begin{tabular}{|c|c|c|c|c|}
\hline & $\begin{array}{l}\text { SpAax-nr sans } \\
\text { signes objectifs }\end{array}$ & $\begin{array}{l}\text { SpAax avec des } \\
\text { signes objectifs }\end{array}$ & Tous les patients & $\mathbf{P}^{*}$ \\
\hline $\begin{array}{l}\text { Age à l'initiation des anti-TNF, moyenne } \\
\text { (années) }\end{array}$ & $42.4(10.9), \mathrm{N}=84$ & $42.5(11.3), N=84$ & $42.5(11.1)$ & 0.934 \\
\hline $\begin{array}{l}\text { Durée des symptômes, moyenne } \\
\text { (années) }\end{array}$ & $10.2(8.9), N=70$ & $11(8.2), \mathrm{N}=76$ & $10.6(8.5)$ & 0.605 \\
\hline Femme & $64 / 84(76.2 \%)$ & $64 / 84(76.2 \%)$ & $128 / 168(76.2 \%)$ & $\mathrm{N} / \mathrm{A}$ \\
\hline IMC, moyenne $\left(\mathrm{kg} / \mathrm{m}^{2}\right)$ & $25.7(5.4), N=69$ & $25.2(5.5), \mathrm{N}=59$ & $25.5(5.4)$ & 0.669 \\
\hline Fumeurs actifs & $31 / 66(47 \%)$ & $26 / 59(44.1 \%)$ & $57 / 125(45.6 \%)$ & 0.442 \\
\hline Dépression & $14 / 80(17.5 \%)$ & $12 / 84(14.3 \%)$ & $26 / 164(15.9 \%)$ & 0.363 \\
\hline Traitement anti-dépresseur & $20 / 79(25.3 \%)$ & $13 / 84(15.5 \%)$ & $33 / 163(20.2 \%)$ & 0.086 \\
\hline Sacro-iliite radiographique & $0 / 84(0 \%)$ & $40 / 83(48.2 \%)$ & $40 / 167(24 \%)$ & $<0.0001$ \\
\hline Sacro-iliite à l'IRM & $0 / 80(0 \%)$ & $38 / 55(69.1 \%)$ & $38 / 135(28.1 \%)$ & $<0.0001$ \\
\hline CRP élevée (> $5 \mathrm{mg} / \mathrm{L}$ ) & $0 / 84(0 \%)$ & $36 / 84(42.9 \%)$ & $36 / 168(21.4 \%)$ & $<0.0001$ \\
\hline Taux de CRP, moyenne (mg/L) & $2(0), N=84$ & $13.1(16.7), N=82$ & $7.5(12.9)$ & $<0.0001$ \\
\hline \multicolumn{5}{|l|}{ En cours } \\
\hline - MII sous traitement & $0 / 84(0 \%)$ & $10 / 84(11.9 \%)$ & $10 / 168(6 \%)$ & 0.001 \\
\hline - psoriasis & $4 / 84(4.8 \%)$ & $6 / 84(7.1 \%)$ & $10 / 168(6 \%)$ & 0.746 \\
\hline - arthrite & $0 / 84(0 \%)$ & $7 / 84(8.3 \%)$ & $7 / 168(4.2 \%)$ & 0.007 \\
\hline - uvéite & $0 / 84(0 \%)$ & $2 / 84(2.4 \%)$ & $2 / 168(1.2 \%)$ & 0.249 \\
\hline - dactylite & $0 / 84(0 \%)$ & $3 / 84(3.6 \%)$ & $3 / 168(1.8 \%)$ & 0.123 \\
\hline - enthésite & $0 / 84(0 \%)$ & $0 / 84(0 \%)$ & 0/168 (0\%) & $\mathrm{N} / \mathrm{A}$ \\
\hline Lombalgie inflammatoire & $84 / 84(100 \%)$ & $84 / 84(100 \%)$ & $168 / 168(100 \%)$ & $\mathrm{N} / \mathrm{A}$ \\
\hline Bonne réponse aux AINS & $63 / 84(75 \%)$ & $61 / 80(76.3 \%)$ & $124 / 164(75.6 \%)$ & 0.499 \\
\hline Histoire familiale de SpA & $26 / 84(31 \%)$ & $16 / 84(19 \%)$ & $42 / 168(25 \%)$ & 0.054 \\
\hline \multicolumn{5}{|l|}{ Antécédents de } \\
\hline - CRP élevée & $2 / 84(2.4 \%)$ & $41 / 84(48.8 \%)$ & $43 / 168(25.6 \%)$ & $<0.0001$ \\
\hline - MII & $3 / 84(3.6 \%)$ & $8 / 84(9.5 \%)$ & $11 / 168(6.5 \%)$ & 0.211 \\
\hline -psoriasis & $4 / 84(4.8 \%)$ & $3 / 84(3.6 \%)$ & $7 / 168(4.2 \%)$ & 1 \\
\hline - arthrite & $1 / 84(1.2 \%)$ & $9 / 84(10.7 \%)$ & $10 / 168(6 \%)$ & 0.018 \\
\hline - uvéite & $6 / 84(7.1 \%)$ & $17 / 84(20.2 \%)$ & $23 / 168(13.7 \%)$ & 0.023 \\
\hline - dactylite & $1 / 84(1.2 \%)$ & $2 / 84(2.4 \%)$ & $3 / 168(1.8 \%)$ & 1 \\
\hline - enthésite & $0 / 84(0 \%)$ & $0 / 84(0 \%)$ & $0 / 168(0 \%)$ & $\mathrm{N} / \mathrm{A}$ \\
\hline Traitement en cours par AINS & $43 / 84(51.2 \%)$ & $39 / 83(47 \%)$ & $82 / 167(49.1 \%)$ & 0.430 \\
\hline Corticothérapie en cours & $3 / 84(3.6 \%)$ & $5 / 84(6 \%)$ & $8 / 168(4.8 \%)$ & 0.360 \\
\hline Traitement en cours par DMARD & $3 / 84(3.6 \%)$ & $6 / 84(7.1 \%)$ & $9 / 168(5.4 \%)$ & 0.496 \\
\hline \multicolumn{5}{|l|}{ Anti-TNF : } \\
\hline ETN & $43 / 84(51.2 \%)$ & $43 / 84(51.2 \%)$ & $86 / 168(51.2 \%)$ & \multirow{5}{*}{0.814} \\
\hline IFX & $18 / 84(21.4 \%)$ & $20 / 84(23.8 \%)$ & $38 / 168(22.6 \%)$ & \\
\hline ADA & $17 / 84(20.2 \%)$ & $16 / 84(19 \%)$ & $33 / 168(19.6 \%)$ & \\
\hline CZP & $1 / 84(1.2 \%)$ & $0 / 84(0 \%)$ & $1 / 168(0.6 \%)$ & \\
\hline Golimumab & $5 / 84(6 \%)$ & $5 / 84(6 \%)$ & $10 / 168(6 \%)$ & \\
\hline $\begin{array}{l}\text { Score BASDAI }(0 \text { - 10) à l'initiation des } \\
\text { anti-TNF, moyenne }\end{array}$ & $6.1(1.4), \mathrm{N}=84$ & $5.6(1.5), \mathrm{N}=84$ & $5.9(1.5)$ & 0.069 \\
\hline
\end{tabular}

Toutes les données sont exprimées en moyenne \pm ET pour les variables continues ou sous forme de nombre (\%) pour les variables catégorielles.

* comparaison entre les 2 groupes, SpAax-nr sans signes objectifs contre SpAax avec des signes objectifs

ADA : Adalimumab, SpAax : spondyloarthrite axiale, BASDAI : Bath Ankylosing Spondylitis Disease Activity Index, IMC : indice de masse corporelle, CRP : protéine C-réactive, CZP : Certolizumab pegol, DMARD : disease-modifying antirheumatic drugs, ETN : Etanercept, HLA B 27 : human leukocyte antigen B 27, MII : 
maladie inflammatoire de l'intestin, IFX : Infliximab, IRM : imagerie par résonance magnétique, $\mathrm{N}:$ nombre de patients avec données disponibles, NA : non applicable, SpAax-nr : spondyloarthrite axiale non-radiographique, AINS : anti-inflammatoires non stéroïdiens, anti-TNF : inhibiteurs du TNF. 
Tableau 2 : Facteurs associés à la réponse BASDAI 50 à 3 mois et sur une année dans la population globale, en analyse univariée

\begin{tabular}{|c|c|c|c|c|c|c|}
\hline & \multicolumn{3}{|c|}{ Population globale à 3 mois } & \multicolumn{3}{|c|}{ Population globale sur 1 an } \\
\hline & Répondeurs & Non-répondeurs & $\mathrm{p}$ & Répondeurs & Non-répondeurs & $\mathrm{p}$ \\
\hline $\begin{array}{l}\text { Age à l'initiation des anti- } \\
\text { TNF, moyenne (années) }\end{array}$ & $39.7(10), \mathrm{N}=48$ & $43.3(11.3), \mathrm{N}=114$ & 0.054 & $40.8(11.2), \mathrm{N}=70$ & $40.8(11.2), \mathrm{N}=98$ & 0.108 \\
\hline $\begin{array}{l}\text { Durée des symptômes, } \\
\text { moyenne (années) }\end{array}$ & $10.1(8.4), N=46$ & $10.9(8.7), N=97$ & 0.609 & $10.3(8.2), \mathrm{N}=67$ & $10.9(8.8), N=79$ & 0.667 \\
\hline Femme & $34 / 48(70.8 \%)$ & $90 / 114(78.9 \%)$ & 0.181 & $51 / 70(72.9 \%)$ & $77 / 98(78.6 \%)$ & 0.249 \\
\hline Fumeurs actifs & $17 / 33(51.5 \%)$ & $37 / 87$ (42.5\%) & 0.249 & $22 / 47$ (46.8\%) & $35 / 78$ (44.9\%) & 0.489 \\
\hline IMC, moyenne $\left(\mathrm{kg} / \mathrm{m}^{2}\right)$ & 24.0 (4.6), $N=33$ & 26.1 (5.7), $\mathrm{N}=89$ & 0.061 & $24.2(4.6), N=47$ & 26.2 (5.7), $N=81$ & 0.039 \\
\hline Obésité (IMC $\geq 30$ kg/m²) & $5 / 33(15.2 \%)$ & $22 / 89(24.7 \%)$ & 0.189 & $6 / 47(12.8 \%)$ & $21 / 81(25.9 \%)$ & 0.06 \\
\hline $\begin{array}{l}\text { Surpoids ou obésité } \\
\left(\mathrm{IMC} \geq 25 \mathrm{~kg} / \mathrm{m}^{2} \text { ) }\right.\end{array}$ & $11 / 33(33.3 \%)$ & $46 / 89(51.7 \%)$ & 0.054 & $17 / 47(36.2 \%)$ & $43 / 81(53.1 \%)$ & 0.048 \\
\hline Dépression & $6 / 48(12.5 \%)$ & $17 / 110(15.5 \%)$ & 0.414 & $8 / 69(11.6 \%)$ & $18 / 95(18.9 \%)$ & 0.145 \\
\hline $\begin{array}{l}\text { Traitement anti- } \\
\text { dépresseur }\end{array}$ & $8 / 48(16.7 \%)$ & $23 / 109$ (21.1\%) & 0.341 & $10 / 69(14.5 \%)$ & $23 / 94(24.5 \%)$ & 0.084 \\
\hline $\begin{array}{l}\text { Sacro-iliite } \\
\text { radiographique }\end{array}$ & $16 / 47(34 \%)$ & $22 / 114(19.3 \%)$ & 0.038 & $25 / 69(36.2 \%)$ & $15 / 98(15.3 \%)$ & 0.002 \\
\hline Sacro-iliite à l'IRM & $19 / 33(57.6 \%)$ & 18/97 (18.6\%) & $<0.0001$ & $22 / 47(46.8 \%)$ & $16 / 88(18.2 \%)$ & 0.001 \\
\hline CRP élevée (> $5 \mathrm{mg} / \mathrm{L}$ ) & $16 / 48(33.3 \%)$ & $20 / 114(17.5 \%)$ & 0.025 & $21 / 70(30 \%)$ & $15 / 98(15.3 \%)$ & 0.018 \\
\hline $\begin{array}{l}\text { Taux de CRP, moyenne } \\
(\mathrm{mg} / \mathrm{L})\end{array}$ & $10.6(15.1), \mathrm{N}=47$ & $6.44(12.0), \mathrm{N}=113$ & 0.066 & $10.3(16), N=69$ & $5.4(9.8), \mathrm{N}=97$ & 0.016 \\
\hline $\begin{array}{l}\text { En cours } \\
\text { - MII sous traitement } \\
\text { - psoriasis }\end{array}$ & $\begin{array}{l}3 / 48(6.3 \%) \\
1 / 48(2.1 \%)\end{array}$ & $\begin{array}{l}7 / 114(6.1 \%) \\
9 / 114(7.9 \%)\end{array}$ & $\begin{array}{l}0.613 \\
0.147\end{array}$ & $\begin{array}{l}5 / 70(7.1 \%) \\
4 / 70(5.7 \%)\end{array}$ & $\begin{array}{l}5 / 98(5.1 \%) \\
6 / 98(6.1 \%)\end{array}$ & $\begin{array}{l}0.407 \\
0.593\end{array}$ \\
\hline - arthrite & $3 / 48(6.3 \%)$ & $4 / 114(3.5 \%)$ & 0.343 & $3 / 70(4.3 \%)$ & 4/98 (4.1\%) & 0.619 \\
\hline - uvéite & $0 / 48(0 \%)$ & $1 / 114(0.9 \%)$ & 0.704 & $0 / 70(0 \%)$ & $2 / 98(2 \%)$ & 0.339 \\
\hline - dactylite & $2 / 48(4.2 \%)$ & $1 / 114(0.9 \%)$ & 0.210 & $2 / 70(2.9 \%)$ & $1 / 98(1 \%)$ & 0.375 \\
\hline Bonne réponse aux AINS & $39 / 47(83 \%)$ & $80 / 111(72.1 \%)$ & 0.104 & $54 / 67(80.6 \%)$ & $70 / 97$ (72.2\%) & 0.146 \\
\hline Histoire familiale de SpA & $8 / 48(16.7 \%)$ & $32 / 114(28.1 \%)$ & 0.088 & $14 / 70(20 \%)$ & $28 / 98(28.6 \%)$ & 0.278 \\
\hline $\begin{array}{l}\text { Antécédents de } \\
\text { - CRP élevée } \\
\text { - MII }\end{array}$ & $\begin{array}{l}16 / 48(33.3 \%) \\
3 / 48(6.3 \%)\end{array}$ & $\begin{array}{l}27 / 114(23.7 \%) \\
8 / 114(7 \%)\end{array}$ & $\begin{array}{l}0.141 \\
0.581\end{array}$ & $\begin{array}{l}24 / 70(34.3 \%) \\
6 / 70(8.6 \%)\end{array}$ & $\begin{array}{l}19 / 98(19.4 \%) \\
5 / 98(5.1 \%)\end{array}$ & $\begin{array}{l}0.023 \\
0.278\end{array}$ \\
\hline -psoriasis & $0 / 48(0 \%)$ & $7 / 114(6.1 \%)$ & 0.105 & $2 / 70(2.9 \%)$ & $5 / 98(5.1 \%)$ & 0.381 \\
\hline - arthrite & $6 / 48(12.5 \%)$ & $4 / 114(3.5 \%)$ & 0.066 & 7/70 (10\%) & 3/98 (3.1\%) & 0.062 \\
\hline - uvéite & $10 / 48(20.8 \%)$ & $12 / 114(10.5 \%)$ & 0.070 & $12 / 70(17.1 \%)$ & $11 / 98(11.2 \%)$ & 0.191 \\
\hline - dactylite & $2 / 48(4.2 \%)$ & $1 / 114(0.9 \%)$ & 0.210 & $3 / 70(4.3 \%)$ & $0 / 98(0 \%)$ & 0.071 \\
\hline $\begin{array}{l}\text { Traitement en cours par } \\
\text { AINS }\end{array}$ & $28 / 48(58.3 \%)$ & $50 / 114(43.9 \%)$ & 0.184 & $37 / 69(53.6 \%)$ & $45 / 98(45.9 \%)$ & 0.306 \\
\hline $\begin{array}{l}\text { Traitement en cours par } \\
\text { DMARD }\end{array}$ & $1 / 48(2.1 \%)$ & $7 / 114(6.1 \%)$ & 0.256 & $2 / 70(2.9 \%)$ & 7/98 (7.1\%) & 0.195 \\
\hline Anti-TNF : & & & & & & \\
\hline $\begin{array}{l}\text { ETN } \\
\text { JFX }\end{array}$ & $32 / 48(66.7 \%)$ & $51 / 114(44.7 \%)$ & & $42 / 70(60 \%)$ & $44 / 98$ (44.9\%) & \\
\hline ADA & $\begin{array}{l}8 / 48(16.7 \%) \\
6 / 48(12.5 \%)\end{array}$ & $\begin{array}{l}30 / 114(26.3 \%) \\
24 / 114(21.1 \%)\end{array}$ & 0.072 & $\begin{array}{l}16 / 70(22.9 \%) \\
9 / 70(12.9 \%)\end{array}$ & $\begin{array}{l}22 / 98(22.5 \%) \\
24 / 98(24.5 \%)\end{array}$ & 0.148 \\
\hline $\begin{array}{l}\text { CZP } \\
\text { Golimumab }\end{array}$ & $0 / 48(0 \%)$ & $1 / 114(0.9 \%)$ & & $0 / 70(0 \%)$ & $1 / 98(1 \%)$ & \\
\hline $\begin{array}{l}\text { Score BASDAI }(0 \text { - 10) à } \\
\text { l'initiation des anti-TNF, } \\
\text { moyenne }\end{array}$ & $5.4(1.5), \mathrm{N}=48$ & $6.0(1.5), \mathrm{N}=114$ & 0.017 & $5.6(1.4), \mathrm{N}=70$ & $6.1(1.5), \mathrm{N}=98$ & 0.023 \\
\hline
\end{tabular}




\begin{tabular}{|c|c|c|c|c|c|c|}
\hline $\begin{array}{l}\text { Score BASDAI (0 - 10) à } 3 \\
\text { mois, moyenne }\end{array}$ & $1.6(0.9), \mathrm{N}=46$ & $5.1(1.6), N=108$ & $<0.0001$ & $2.3(1.6), \mathrm{N}=66$ & $5.4(1.5), \mathrm{N}=88$ & $<0.0001$ \\
\hline Score BASDAI (0 - 10) à 6 & & & & & & \\
\hline mois, moyenne & $2.0(1.6), N=36$ & $5.1(2.4), \mathrm{N}=73$ & $<0.0001$ & $2.1(1.4), \mathrm{N}=57$ & $6.0(1.9), \mathrm{N}=58$ & $<0.0001$ \\
\hline $\begin{array}{l}\text { Score BASDAI }(0 \text { - 10) à } \\
\text { un an, moyenne }\end{array}$ & $2.3(1.8), \mathrm{N}=37$ & $4.6(2.2), \mathrm{N}=46$ & $<0.0001$ & $2.5(1.8), \mathrm{N}=56$ & $5.6(1.7), \mathrm{N}=30$ & $<0.0001$ \\
\hline $\begin{array}{l}\text { Répondeurs BASDAI } 50 \text { à } \\
\text { chaque visite }\end{array}$ & NA & NA & NA & $26 / 70(37,1 \%)$ & NA & NA \\
\hline
\end{tabular}

ADA: Adalimumab, SpAax : spondyloarthrite axiale, BASDAI : Bath Ankylosing Spondylitis Disease Activity Index, IMC : indice de masse corporelle, CRP : protéine C-réactive, CZP : Certolizumab pegol, DMARD : disease-modifying antirheumatic drugs, ETN : Etanercept, HLA B 27 : human leukocyte antigen B 27, MII : maladie inflammatoire de l'intestin, IFX : Infliximab, IRM : imagerie par résonance magnétique, $\mathrm{N}:$ nombre de patients avec données disponibles, NA : non applicable, SpAax-nr : spondyloarthrite axiale non-radiographique, AINS : anti-inflammatoires non stéroïdiens, anti-TNF : inhibiteurs du TNF. 